\title{
Genetic Diversity of Eggplant Revealed by SSR Markers
}

\author{
Wang Qiu-jin ${ }^{1,2}$, \\ 1.Department of Biotechnology \\ Beijing University of Agriculture \\ Beijing , P.R.China \\ 2. College of Horticulture \\ Xinjiang Agricultural University \\ Urumchi 830052, P.R.China \\ E-mail:wangqiujin_2001@163.com
}

\author{
Zhao Fu-kuan*, Sun Qing-Peng, Yang Ai-zhen \\ Department of Biotechnology \\ Beijing University of Agriculture \\ Beijing, P.R.China \\ E-mail for corresponding author: \\ zhaofukuan@sina.com
}

\begin{abstract}
Eighty eight accessions including eighty six of Solanum melongena and two wild related species (Solanum integrifolium and Solanum torvum) were used to analyze the genetic variation with 23 SSR primers. And 20 primers were successfully amplified, of which 19 showed polymorphism with a total of 68 detected alleles. The number of alleles per primer ranged from 2 to 6, with a mean of 3.6. Among 19 polymorphism primers, four primer pairs (EM117, EM155, EM126, EM127) gave rise to multiple polymorphic bands, two of which could be used for varieties certification of eggplant. The average genetic diversity of all accessions measured by the Nie's genetic diversity, Shannon's Information index and Effective number of alleles were 0.41 (range $0.12 \sim 0.80$ ), 0.7443 (rang from $0.2617 \sim 1.6958$ ) and 2.0151(ranged from 1.1307 5.310), respectively. Genetic similarity among all the accessions ranged from 0.09 to 1 with an average of 0.69 . The high diversity was displayed. Additionally, $S$. melongena had high average similarity with the wild species $S$. integrifolium $(0.61)$ and low average similarity with $S$. torvum (0.26). UPGMA clustering at genetic similarity value of 0.60 grouped all the eggplant accessions into four groups, where S.torvum formed a separate and more distant cluster. Moreover, the fourth group can be further classified into two sub-groups, and the wild species $S$. integrifolium can be clearly separated from the other cultivated eggplants. However, majority of the cultivated eggplants were not clustered in ways concordant with taxonomic information such as collection sites, fruit shape or any other known trait. The genetic diversity and phylogenetic relationships of eggplant accessions reported in this study might be used for breeder when planning future crosses between cultivated eggplant and the wild species.

Keywords- Genetic diversity, SSR markers, Eggplant, Cluster analysis.
\end{abstract}

\section{INTRODUCTION}

Eggplant (Solanum melongena L.), known as aubergine or brinjal, belongs to the Solanaceae. The exact origin of eggplant is uncertain; however, it may have been indirectly derived from the wild African species S.incanum [1] and was domesticated in India and southeast China. Today, China accumulates a rich eggplant germplasm resource as well as its wide relatives in its long cultivated history. The majority of wild species are useful source of resistance against diseases available for cultivated eggplant improvement. Knowledge of the genetic variation of eggplant is important for their efficient use in breeding programs and for conservation purposes. Molecular marker analysis, joined to phenotypic evaluation, is a powerful tool for grouping of genotypes based on genetic similarity data, and for selection of progenitors that might constitute new breeding populations. And as Westman et al. [2] puts, this tool is also very useful for identification and management of germplasm collections. However, little has been done to assess genetic diversity of eggplant by using molecular markers. And only a few research papers published concerning with RAPD [3;4;5;6;7] , AFLP[8;9], ISSR [10], and SSR [11;12] markers.Of the several classes of DNA-based markers, the microsatellites (or simple sequence repeat SSR) are polymerase chain reaction (PCR)-based, highly polymorphic, multi-allelic, frequently codominant, highly reproducible and randomly and widely distributed in the genome [13]. It has been recently developed and proved to be useful for accession discrimination and assessment of genetic variation $[14 ; 15]$. Moreover, since little genetic variability has been detected in cultivated eggplant $[3 ; 11 ; 16 ;]$, the use of a polymorphic marker, such as SSRs, in addition to distinguishing closely related genotypes, should also be useful for phylogenetic studies, as demonstrated in potato [17], tomato [18] and wheats [19].

Here, we developed SSR markers for genetic analysis in eggplant: (1) to analysis their amplify genotype and genetic diversity, (2) to assess genetic relationship among cultivated eggplant (S.melogena) and the wild species.

\section{MATERIALS AND METHODS}

Plant materials:86 cultivated eggplants, as well as two wild species $S$. integrifolium and $S$. torvum, were used for analyzing the genetic diversity.

DNA Extraction: Genomic DNA was isolated from fresh leave of each individual using CTAB method [20] and was purified through additional steps. The extracted DNA was diluted to 50 ng $\mu \mathrm{L}^{-1}$ with TE buffer and stored at $-20^{\circ} \mathrm{C}$ for PCR amplification. DNA quality was examined by electrophoresis in $0.8 \%$ agarose gel.

SSR amplifications:Twenty three SSR primer pairs(EM104,EM107,EM114,EM116,EM117,EM119,EM120, EM126,EM127,EM128,EM131,EM133,EM134,EM135,EM1

* Correspondence Zhao Fu-kuan, Professor,Ph D 
39,EM140,EM141,EM145,EM146,EM151,EM155,EM157,E M162)previously developed for eggplant[11] were carried on the DNA amplification. The PCR was set up as follows: A total volume of $20 \mu \mathrm{l}$ contained $20 \mathrm{ng}$ templates DNA, 1× reaction buffer, $1.5 \mathrm{mM} \mathrm{MgCl}_{2}, 0.2 \mathrm{mM}$ dNTPs, $0.2 \mu \mathrm{mol} \mathrm{L}^{-1}$ each of forward and reverse primer and $0.5 \mathrm{U}$ Taq DNA polymerase. The reaction was performed in a Thermo HBPEX02 thermo cycler (Thermo USA), and the programmer was as follows: an initial denaturation cycle of 5 min at $94^{\circ} \mathrm{C}$, followed by 35 cycles of $94^{\circ} \mathrm{C}$ for $0.5 \mathrm{~min}, 60$ or $65^{\circ} \mathrm{C}$ for $1 \mathrm{~min}, 72^{\circ} \mathrm{C}$ for $1.5 \mathrm{~min}$ and finally a 5 min extension at $72^{\circ} \mathrm{C}$. The PCR products were stored at $4^{\circ} \mathrm{C}$ until loading.

Electrophoresis:The PCR reaction was mixed with half volume of formamide loading-buffer (98\% formamide deionized, $10 \mathrm{mM}$ of EDTA (pH 8.0), 0.25\% bromophenol blue, and $0.25 \%$ xylene cyanol FF), then heated at $94^{\circ} \mathrm{C}$ for 5 min, $5 \mu \mathrm{L}$ of the denaturing mixture was loaded and separated on $5 \%(\mathrm{w} / \mathrm{v})$ denaturing polyacrilamide gels containing $7.5 \mathrm{M}$ urea and $30 \%$ formamide in $1 \times \mathrm{TBE}$ buffer at a constant current $70 \mathrm{~mA}$ for $1 \mathrm{~h}$. The DNA bands were visualized by silver staining according to the protocol described by Brant et al. [21]. The sizes of the alleles were estimated by comparison to Puc19 DNA/Msp I (HpaII) marker (MBI, Fermentas).

Data analysis: The observing fragment data were inputted in a computer file as a binary matrix, where 0 and 1 coded for absence and presence of a band. NTSYSpc-2.10e and POPGENE 1.32 [22] software were used for data analysis.

The genetic diversity analysis was estimated with parameters calculated using POPGENE. The genetic relationships among the 88 eggplant accessions were investigated using the NTSYS-pc2.10e program. Dice similarity coefficients generate from SIMQUAL (similarity for qualitative date) routine, and then were used to construct dendrograms using the UPGMA (unweighted pair group method with arithmetic average) method, from SAHN (sequential agglomerative hierarchical and nested clustering) routine.

\section{RESULTS AND ANALYSIS}

Fragments analysis of the SSR amplification loci :In the present study, among 23 SSR primers, two primers (EM 104, EM 135) failed to amplify the expected PCR fragments, one primer (EM 162) amplified monomorphic banding patterns, while the other primer (EM 139) amplified too many useless fragments, even after several attempts had been made with these primer pairs. The remaining 19 primers with polymorphism were selected for the further analysis. Among of them 14 primers produced single or two fragments. The rest of five primers (EM 117, EM 119, EM 155, EM 126 and EM 127) gave rise to 3-4 fragments or band complex pattern in all tested species. This is probably due to the amplification of multiple microsatellite-containing loci (for example, in a gene family) using a single pair of primers. The same SSR markers characterization were reported in potato [23]. and peanut [24]. The high levels of polymorphism revealed by such multi-locus microsatellite markers made them extremely powerful tools for differentiating between individual cultivars and accessions [23]. In this study the high level of polymorphism in the selected two primers (EM117, EM155) permit to distinguish the 88 eggplant accessions.

Marker allelic polymorphism:19 polymorphic primers detected 68 alleles in all. The number of alleles per loci ranged from 2 6 with a mean of 3.6, and similar results have been reported by Nunome et al. [11] and Behera et al. [12]. Maximum alleles were 6 detected from EM128 and EM155. The effective number of alleles for each SSR loci ranged from 1.1307 5.3101, with an average of 2.0151. The Nei's Gene diversity $H$ for each SSR ranged from $0.1156 \sim 0.8005$, with an average of 0.4049 . Shannon's Information index (the genotype diversity) was $0.2617 \sim 1.69580$, with an average of 0.7443 .

Genetic relationship:According to the data got from SSR markers, the calculated genetic similarity coefficient among all 88 eggplant accessions ranged from 0.09 (between Jingpinkuaiyuan and $S$. torvum) to 1.00 (between ETC4-03 and ETC4-01) with an average of 0.69. The average similarity coefficient were 0.23 (between $S$. melongena and $S$. torvum), and 0.61 (between $S$. melongena and $S$. Integrifolium). And the later value was close to what Behera et al [12] has reported. A low similarity coefficient, that is 0.25 , occurred between the two weedy species $S$. tovurm and $S$. integrifolium. UPGMA cluster analysis of the similarity data divided all 88 eggplant accessions into four groups at the similarity coefficient of 0.60 .

Group I consisted of only one species, S. torvum (No.41), which is considered as a potential source of resistance to bacterial wilt in eggplant breeding programs.

Group II included two accessions, one inbred lines variety 'Daziguanqie'(No.37) from Dalian, the other common variety "Xinyanerhao"(No.33) from Beijing.

Group III included three accessions, one exotic variety "pusakranti"(No.35) from Holland as well as two inbred lines "Ziguanqie"(No.34) and "Zihongguanqie" (No.40) from Shanxi and Fujian.

Group IV consisted of maximum number of indigenous accessions, a few exotic accessions and the wild species $S$. integrifolium. (No.42) This group can be further classified into two sub-groups:

The first sub-group including only one species, that is the wild species $S$. integrifolium, has a little difference with cultivated eggplant in morphological, growth, and varietals characteristics.

The second sub-group including maximum cultivated eggplant, and was classified into three clusters. The first cluster included two accessions that are LongXinglvqiao (No.59) and Heiyuanshuai (No.70), diversified greatly in shape and color trait. The second cluster included eight accessions, three of them are common varieties, one is near isogenic lines (NIL) and remains are hybrids. The third cluster, including many accessions, were clustered together in a high average similarity coefficient $(>0.5)$ with no relation to collection sites, fruit shape and color, or any other known traits.However, clusters of the majority of the near isogenic lines (NIL) are consistent with known pedigree information. For example, 'ETC4-01'(No.46) and 'ETC4-03' (No.45), 'ETC3-01' (No.56), 'ETC3-02' (No.55)and 'ETC3- 
03' (No.54), and 'ETC6-01' (No.43)and 'ETC6-03(No.45), were classified into smaller cluster respectively; 'HL-01' (No.31) and 'HL-03' (No.32)was clustered together.

\section{DISCUSSIONS:}

In this study, the maximum alleles detected from the prime pairs of EM128 and EM155, while the two loci have a max number of repeat motifs in microsatellites accordingly. A correlation was found between the number of alleles per locus and the number of repeat motifs in microsatellites in this study, which agreed with earlier reports in tomato [18], but contradicted with the reports in eggplant [11]).

The mean Nei's Gene diversity $(H)$ and Shannon's Informatinion index here were 0.4049, and 0.7443, respectively, demonstrated relatively high diversity within the all tested accessions. This agreed with earlier reports in eggplant $[4 ; 8 ; 9 ; 12]$. However, a low diversity was also observed in some studies $[3 ; 10 ; 11]$. Many factors influenced genetic diversity such as population size, population structure, outcrossing, recombination, diversifying selection, background selection and PCR problem and so on. Cui et al. [25] suggests that the degree of polymorphism may correlate with the product of mutation rate and the generation term of the locus. The earliest neutral theory of evolution suggests that the level of polymorphism should be the product of effective population size and the mutation rate. Some data support the connection between selfing rate and level of diversity. Compared with outcrossing species, selfing species often had lower level of diversity. Some studies showed that strong selection pressure is important in decreasing the genetic diversity of some plant species. Many of the grass domesticates have undergone rather modest decreases in diversity relative to their wild relatives [26]. In domesticated maize, the diversity is roughly $30 \%$ below that in its closest wild relative [27]. Lower genetic diversity has been observed in cultivated eggplants than its wild species [11]. Balancing selection and frequency-dependent selection may also play an important role in increasing diversity at specific loci within a genome. For these reasons, the high diversity we observed may result from population size, population structure (our materials including cultivars and wild species come from different sites) and the SSR locus.

The UPGMA cluster analysis utilized in this study showed that S.integrifoluim is more closely related to S.melongena than S.torvum. Similar results were obtained by Chen et al [19].The cross ability relationship among S.melongena cultivars and its related species depicted earlier by NarasimhaRao [12]. It was revealed from his report that the crosses S.melongena $\times$ integrifoluim could produce normal seeds. We support it on molecular level.

The cluster for two wild species were consistent with accepted phylogenies, but most cultivars were not clustered in accord with taxonomic information such as collection sites, fruit shape or any other known trait. The results reported here are agreed with on Ran et al. [7], but contrast to the reported results by Fenlingling et al. [4] who obtained the cluster of 34 eggplant based on fruit shape using RAPD markers. An alternative explanation for the results of classification of 88 eggplants might be the inappropriateness of the SSR markers for resolving relatedness at this higher (interspecific) taxonomic level as Hokanson et al. [28] reported. Some hypotheses for the failure of microsatellites at the inter-specific level have been suggested as follows: restrictions to divergence in the repeat sequences, asymmetries in the mutation process in the repeat regions, and the degradation of the microsatellite regions over time [29], while other possible factors for this failure have been published earlier by other authors [30;31]. Expect above explanation for the cluster of 88 eggplants, another reason may be due in part to the makeup of the collection investigated in this study. Hokanson et al. [28] suggested the RAPD markers have the potential to complement 'classical' taxonomic studies, however larger numbers of different molecular markers and statistical methods should be utilized. In addition, the results suggest that SSR molecular marker also can be used in gene mapping, cultivar certification and marker-assisted breeding of eggplant.

\section{ACKNOWLEDGEMENT}

This work was supported by Funding Project for Academic Human Resources Development in Institutions of Higher Learning Under the Jurisdiction of Beijing Municipality(PHR200907136).

\section{References}

[1]Daunay, M C, Lester R N, Ano G. Cultivated eggplants, in Tropical Plant Breeding, edited by Charrier A., Jacquot M, Hamon S, and Nicolas D. Oxford University Press, Oxford, 2001.200-225

[2]Westman A L, Kresovich S, Use of molecular markers techniques for description of plant genetic variation. In: Plant Genetic Resources: Conservation and Use Edited by: Callow J A, Ford-Lloyd B V, Newbury H J. Wallingford, U K: CAB International, 1997.9-48.

[3] Karihaloo J L, Brauner S, Gottlieb L D. Random amplified polymorphic DNA variation in the eggplant. Theoretical and Applied Genetic, 1995. 90, 767-770.

[4];Feng L L, Dong Y, Lian Y, Jin L P, Wang X W, Song Y. Studies on the genetic relationship in eggplant by RAPD analysis. China Vegetables, 2002.4, 35-36.

[5]Clain C, Silva D D, Fock I, Vaniet S, Carmeille A, Gousset C, Sihachakr D, Luisetti J, Kodja H, Besse P. RAPD genetic homogeneity and high levels of bacterial wilt tolerance in Solanum torvum Sw. (Solanaceae) accessions from Reunion Island. Plant science, 2004.166, 1533-1540

[6]; Chen J, Li H Z, Zhuang T M, Chen H Y. Genetic relationship analysis of cultivated eggplant (Solanum melongena L.) and wild relatives (Solanaceae) with RAPD maker. Journal of Shanghai Jiaotong university (Agricultural Science), 2008.26, 165-167.

[7]Ran J, Song M, Fang C, Wen M L, Liu X J, Liu D C, Li Y J. Analysis of genetic diversity in eggplant (S. melongena L). Southwest China Journal of Agricultural Sciences, 2007.20, 694-697.

[8]Mace E S, Lester R N, Gebhardt C G. AFLP analysis of genetic relationship among the cultivated eggplant Solanum melongena L.and wild relatives (Solanaceae). Theoretical and Applied Genetic, 1999.99, 626-633.

[9]Furini A, Wunder J. Analysis of eggplant (Solanum melongena) -related germplasm: morphological and AFLP data contribute to phylogenetic interpretations and germplasm utilizations. Theoretical and Applied Genetic, 2004.108, 197-208.

[10]Mao W H, Du L M, Bao C L, Hu T H, Zhu Q M, Hu H J. Genetic Diversity and Genetic Relatives Analysis of Southern Long-eggplant Germplasm Based on Inter-simple Sequence Repeat (ISSR). Acta horticulture sinca, 2006.33, 1109-1112 
[11]Nunome T, Suwabe K, Iketani H, Hirai M. Identification and characterization of microsatellites in eggplant. Plant Breeding, 2003.122, 256-262.

[12]Behera T K, Pankaj Sharma, Singh B K, Kumar G, Ravinder Kumar, Mohapatra T, Singh N K. Accessment of genetic diversisty and species relationships in eggplant(Solanum melongena L.)using STMS markers. Scientia Horticulture, 2006.107, 52 357.

[13]Powell W, Morgante M, Andre C, Hanafey M, Vogel J, Tingey S, Rafalasky A. The comparison of RFLP, RAPD, AFLP and SSR (microsatellite) markers for germplasm analysis. Molecular Breeding, 1996.3, 225-238.

[14]Hou CH X, Li M W, Zhang Y H, Qian H Y, Sun P J, Xu A Y, Miao X X, Guo Q H, Xiang H, Huang Y P. Analysis of SSR fingerprints in introduced Silkworm germplasm resources, Agricultural sciences in china, 2007.6, 620-627.

[15]Benor S, Zhang M Y, Wang ZH F, Zhang H S. Assessment of genetic varation in tomato (Solanum lycopersicum L.) inbred lines using SSR molecular markers. Genomics, 2008.35, 373-379.

[16]Doganlar S, Frary A, Daunay M C, Lester R N, Tanksley S D. Conservation of gene function in the Solanaceae as revealed by comparative mapping of domestication traits in eggplant. Genetic, 2002.161, 1713-1726.

[17]Ashkenazi V, Chani E, Lavi U, Levy D, Hillel J, Veilleux R E. Development of microsatellite markers in potato and their use in phylogenetic and fingerprinting analyses. Genome, 2001. 44, 50-62.

[18]He C, Poysa V, Yu K. Development and characterization of simple sequence repeat (SSR) markers and their use in determining relationships among Lycopersicon esculentum cultivars. Theoretical and Applied Genetic, 2003.106, 363-373.

[19]Chen G Y, Li L H. Detection of genetic diversity in synthetic hexaploid wheats using microsatellite markers. Agricultural Sciences in China, 2007. 6, 1403-1410.

[20]Doyle J J, Doyle J L. A rapid total DNA preparation procedure for fresh plant tissue. Focus, 1990.12, 13-15.

[21] Brant J, Bassam, Fast and sensitive silver staining of DNA in polyacrylamide gels. Analytical biochemistry, 1991.196, 80-83.

[22]Yeh F C, Yang R. Microsoft Window-based freeware for population genetic analysis. University of Alberta Tim Boyle, Center for International Forestry Research POPGENE Version 1.31: ftp:// ftp. microsoft.com/ Softlib/ MSLFILES/ HPGL.EXE, 1999.

[23]Provan J, Powell W, Waugh R. Microsatellite analysis of relationships within cultivated potato (Solanum tuberosum). Theoretical applied genetic, 1996.92, 1078-1084.

[24]Tang R H, Gao G Q, He L Q, Han ZH Q, Shan S H, Zhong R C, Zhou C Q, Jiang J, Li Y R, Zhuang W J. Genetic diversity in cultivated groundnut based on SSR markers. Journal of genetic and genomics, 2007.34, 449-459.

[25]Cui X M, Dong Y X, Hou X L, Cheng Y, Zhang J Y, Jin M F. Development and characterization of microsatellite markers in Brassica rapassp. Chinensis and transferability among related species. Agricultural Sciences in China, 2008.7, 19-31

[26]Buckler E S IV, Thornsberry J M, Kresovich S. Molecular diversity, structure and domestication of grasses. Genetical Research, 2001.77: 213-218.

[27]Eyre-Walker A, Gaut R L, Hilton H, Feldman D L, Gaut B S. Investigation of the bottleneck leading to the domestication of maize. Proceedings of the National Academy of Scences of the United States of America, 1998.95, 4441-4446.

[28]Hokanson S C, Lamboy W F, Szewc-Mcfadden A K, Mcferson J R. Microsatellite (SSR) variation in a collection of malus (apple) species and hybids. Euphytica, 2001.118, 281-294.

[29]Goldstein D B, Pollock D D. Launching microsatellites: A review of mutation processes and methods of phylogenetic inference. The Journal of Heredity, 1997. 88, 335-342.

[30]Ellegren H, Primmer C R, Sheldon B C. Microsatellite evolution: Directionality or bias? Nature Genetics, 1995.11, 36-362.

[31] Garza J C, Slatkin M, Freimer N B. Microsatellite allele frequencies in humans and chimpanzees, with implications for constraints on allele size. Molecular Biology and Evolution, 1995.12, 594-603. 